\title{
Boundedness of Solutions of Matrix Nonlinear Volterra Difference Equations
}

\author{
MICHAEL I. GIL ${ }^{a}, *$ and RIGOBERTO MEDINA ${ }^{\mathrm{b}}$ \\ ${ }^{\mathrm{a}}$ Department of Mathematics, Ben Gurion University of the Negev, P.O. Box 653, Beer-Sheva 84105, Israel; ${ }^{\mathrm{b}}$ Departmento de Ciencias Exactas, \\ Universidad de Los Lagos, Casilla 933, Osorno, Chile
}

(Revised 8 February 2001)

\begin{abstract}
Nonlinear discrete-time Volterra equations in a Euclidean space are considered. Conditions for the boundedness of solutions are established by virtue of recent estimates for the norm of the resolvent of Volterra operators. The conditions are formulated in the terms of coefficients of considered equations. In addition, estimates for the $c_{0^{-}}$and $l^{2}$-norms of solutions are derived.
\end{abstract}

Keywords: Volterra difference equations; Boundedness; Estimates for solutions; Matrix

\section{INTRODUCTION}

Volterra difference equations arise in the mathematical modeling of some real phenomena, and also in numerical schemes for solving differential and integral equations, cf. (Kolmanovskii and Myshkis, 1998; Kolmanovskii et al., 2000) and references therein.

One of the basic methods in the theory of stability and boundedness of Volterra difference equations is the direct Lyapunov method ( see (Crisci et al., 1995; Elaydi, 1996; Elaydi and Murakami, 1996) and references therein). But finding of the Lyapunov functionals for Volterra difference equations is a difficult mathematical problem.

In this paper, to establish the boundedness conditions, we will interpret the Volterra equations with matrix kernels as operator equations in appropriate spaces. Such an approach for Volterra difference equations have been used by Kolmanovskii et al. (2000), Kolmanovskii and Myshkis (1998), Kwapisz (1992) and Medina (1996). Our main tool is the recent estimates for the norm of the resolvent of Volterra operators established in Gil' (1995). Under some restriction, these estimates allow us to generalize the main results from the papers of Kolmanovskii et al. (2000), Kolmanovskii and Myshkis (1998), Kwapisz (1992) and Medina (1996). Moreover, we derive inequalities for the $c_{0^{-}}$and $l^{2}$-norms of solutions. Note that our boundedness conditions are formulated in the terms of coefficients of considered equations.

\section{PRELIMINARIES}

Let $C^{n}$ be an $n$-dimensional complex Euclidean space with the Euclidean norm $|\cdot|_{C}$. For a positive $r$, put

$$
\omega_{r}=\left\{h \in C^{n}:|\cdot|_{C^{n}} \leq r\right\}
$$

As usual $c_{0}=c_{0}\left(C^{n}\right)$ is the Banach space of sequences of vectors from $C^{n}$ equipped with the norm

$$
\begin{aligned}
|h|_{c_{0}} & =\sup _{k}\left|h_{k}\right|_{C^{n}}\left(h=\left(h_{k}\right)_{k=1}^{\infty} \in c_{0} \quad\left(h_{k} \in C^{n} ;\right.\right. \\
k & =1,2, \ldots)
\end{aligned}
$$

and $l^{2}=l^{2}\left(C^{n}\right)$ is the Banach space of sequences of vectors from $C^{n}$ equipped with the norm

$$
\begin{aligned}
|h|_{l^{2}} & =\left[\sum_{k=1}^{\infty}\left|h_{k}\right|_{C^{n}}^{2}\right]^{\frac{1}{2}} \\
(h & \left.=\left(h_{k}\right)_{k=1}^{\infty} \in l^{2}, h_{k} \in C^{n}, k=1,2, \ldots\right) .
\end{aligned}
$$

Let $a_{j k}(j, k=1,2, \ldots)$ be $n \times n$-matrices, and $f_{j} \in$ $C^{n} \quad(j=1,2, \ldots)$. Consider the equation

$$
x_{j}=f_{j}+\sum_{k=1}^{j-1} a_{j k}\left[x_{k}+G_{k}\left(x_{k}\right)\right], \quad(j=1,2, \ldots)
$$

*Corresponding author. 
where the mappings $G_{k}: C^{n} \rightarrow C^{n}$ have the property

$$
\begin{aligned}
& \left.\left|G_{k}\right|(h)\right|_{C^{n}} \leq q_{k}|h|_{C^{n}} \\
& \quad\left(h \in \omega_{r} ; k=1,2, \ldots ; r \leq \infty\right) .
\end{aligned}
$$

Rewrite Eq. (1) as

$$
x=f+V x+F(x),
$$

where $f=\left(f_{k}\right), V$ and $F$ are defined in $c_{0}$ by

$$
[V x]_{j}=\sum_{k=1}^{j-1} a_{j k} x_{k}
$$

and

$$
[F x]_{j}=\sum_{k=1}^{j-1} a_{j k} G_{k}\left(x_{k}\right) .
$$

Here $[h]_{j}$ means the $j$-th coordinate of the element $h \in$ $c_{0}$.

Let $N(A)$ be the Hilbert-Schmidt (Frobenius) norm of a matrix $A$. That is, $N^{2}(A)=\operatorname{Trace}\left(A A^{*}\right)$. It is assumed that

$$
\begin{gathered}
N(V) \equiv\left[\sum_{j=1}^{\infty} \sum_{k=1}^{j-1}\left|a_{j k}\right|_{C^{n}}^{2}\right]^{\frac{1}{2}}<\infty \\
|f|_{l^{2}} \equiv\left[\sum_{k=1}^{\infty}\left|f_{k}\right|_{C^{n}}^{2}\right]^{\frac{1}{2}}<\infty
\end{gathered}
$$

and

$$
N_{q} \equiv\left[\sum_{j=1}^{\infty} \sum_{k=1}^{j-1}\left|a_{j k}\right|_{C^{n}}^{2} q_{k}^{2}\right]^{\frac{1}{2}}<\infty .
$$

Clearly, if $q_{k} \leq b_{0}<\infty(k=1,2, \ldots)$, then Eq. (4) implies $N_{q} \leq b_{0} N(V)$.

Furthermore, denote

$$
m(V)=\sum_{k=0}^{\infty} \frac{N^{k}(V)}{\sqrt{k !}} .
$$

By the Schwarz inequality it is not hard to show that

$$
m(V) \leq \sqrt{2} \exp \left[N^{2}(V)\right] .
$$

\section{THE MAIN RESULT}

Lemma 1. Let condition (2) hold with $r=\infty$. Then under the conditions (4-6), and

$$
m(V) N_{q}<1
$$

a solution $x=\left(x_{k}\right)$ of Eq. (1) is in $l^{2}$. Moreover, it satisfies the inequality

$$
|x|_{l^{2}} \leq m(V)\left(1-m(V) N_{q}\right)^{-1}|f|_{l^{2}} .
$$

Proof. By Theorem 2.4.1 from Gil' (1995),

$$
\left|(I-V)^{-1}\right|_{l^{2}} \leq m(V)
$$

Moreover, due to Eq. (2) and the Schwarz inequality

$$
\begin{aligned}
|F(x)|_{l^{2}}^{2} & =\sum_{j=1}^{\infty}\left|\sum_{k=1}^{j-1} a_{j k} G_{k}\left(x_{k}\right)\right|_{C^{n}}^{2} \\
& \leq \sum_{j=1}^{\infty}\left[\sum_{k=1}^{j-1}\left|a_{j k}\right|_{C^{n}} q_{k}\left|x_{k}\right|_{C^{n}}\right]^{2} \\
& \leq \sum_{j=1}^{\infty} \sum_{k=1}^{j-1}\left|a_{j k}\right|_{C^{n}}^{2} q_{k}^{2-1} \sum_{k=1}^{j-1}\left|x_{k}\right|_{C^{n}}^{2} .
\end{aligned}
$$

Hence,

$$
|F(x)|_{l^{2}} \leq N_{q}|x|_{l^{2}} .
$$

Rewrite Eq. (3) as

$$
x=(I-V)^{-1}(f+F(x)) .
$$

From Eqs. (9) and (10), it follows that

$$
|x|_{l^{2}} \leq m(V)\left[|f|_{l^{2}}+N_{q}|x|_{l^{2}}\right] .
$$

Thus, by Eq. (7) we get Eq. (8), concluding the proof. Note that the comparison theorems for Volterra difference equations of the type Eq. (1) were established in Medina (1999).

Put

$$
M_{1}(V, q)=\max _{j}\left[\sum_{k=1}^{j-1}\left|\left(1+q_{k}\right) a_{j k}\right|_{C^{n}}^{2}\right]^{\frac{1}{2}}
$$

and

$$
c(f)=|f|_{c_{0}}+|f|_{l^{2}} M_{1}(V, q) m(V)\left(1-m(V) N_{q}\right)^{-1} .
$$

Lemma 2. Under the hypotheses of Lemma 1, the estimate

$$
|x|_{c_{0}} \leq c(f)
$$

is valid.

Proof. By Eqs. (1) and (2),

$$
|x|_{c_{0}} \leq|f|_{c_{0}}+\max _{j} \sum_{k=1}^{j-1}\left|a_{j k}\right|_{C^{n}}\left(1+q_{k}\right)\left|x_{k}\right|_{C^{n}} .
$$


The Schwarz inequality yields

$$
\begin{aligned}
|x|_{c_{0}} & \leq|f|_{c_{0}}+\max _{j}\left[\sum_{k=1}^{j-1}\left|\left(1+q_{k}\right) a_{j k}\right|_{C^{n}}^{2} \sum_{k=1}^{j-1}\left|x_{k}\right|^{2}\right]^{\frac{1}{2}} \\
& \leq|f|_{c_{0}}+M_{1}(V, q)|x|_{l^{2}} .
\end{aligned}
$$

From Lemma 1 we infer that

$|x|_{c_{0}} \leq|f|_{c_{0}}+m(V)\left(1-m(V) N_{q}\right)^{-1}|f|_{l^{2}} M_{1}(V, q)=c(f)$

concluding the proof.

By a simple application of the Uryshon lemma and Lemma 2 we get the main result of the paper

Theorem 1. Under conditions (2) and (4-7), let

$$
c(f) \leq r
$$

Then, a solution $x=\left(x_{k}\right)$ of Eq. (1) is bounded. Moreover, the inequality Eq. (12) is valid.

\section{PARTICULAR CASES}

Let us consider the linear system with disturbances

$$
x_{j}=f_{j}+\sum_{k=1}^{j-1}\left(a_{j k}+b_{j k}\right) x_{k} ; \quad j, k=1,2, \ldots
$$

where $\left(a_{j k}\right)$ and $\left(b_{j k}\right)$ are $n \times n$-matrices, and $f_{j} \in C^{n}$ $(j, k=1,2, \ldots)$. Rewrite Eq. (14) as

$$
x=f+V x+W x
$$

where $f=\left(f_{k}\right), V$ and $W$ are defined in $c_{0}$ by

$$
[V x]_{j}=\sum_{k=1}^{j-1} a_{j k} x_{k}
$$

and

$$
[W x]_{j}=\sum_{k=1}^{j-1} b_{j k} x_{k}
$$

It is assumed that

$$
N(W)=\left[\sum_{j=1}^{\infty} \sum_{k=1}^{j-1}\left|b_{j k}\right|_{C^{n}}^{2}\right]^{\frac{1}{2}}<\infty .
$$

Lemma 1 implies

Corollary 1. Under conditions (4), (5) and (16), let

$$
m(V) N(W)<1
$$

Then a solution $x=\left(x_{k}\right)$ of Eq. (14) is in $l^{2}$. Moreover, it satisfies the inequality

$$
|x|_{l^{2}} \leq m(V)(1-m(V) N(W))^{-1}|f|_{l^{2}} .
$$

Furthermore, put

$$
\begin{aligned}
& M_{2}(V)=\max _{j}\left[\sum_{k=1}^{j-1}\left|a_{j k}\right|_{C^{n}}^{2}\right]^{\frac{1}{2}}, \\
& M_{3}(W)=\max _{j}\left[\sum_{k=1}^{j-1}\left|b_{j k}\right|_{C^{n}}^{2}\right]^{\frac{1}{2}},
\end{aligned}
$$

and

$$
\begin{aligned}
c_{1}(f)= & |f|_{c_{0}}+m(V)\left(M_{2}(V)+M_{3}(W)\right)(1 \\
& -m(V) N(W))^{-1}|f|_{l^{2}}
\end{aligned}
$$

Lemma 2 implies

Corollary 2. Under the hypotheses of Corollary 1, for a solution of Eq. (14) the estimate

$$
|x|_{c_{0}} \leq c_{1}(f)
$$

is valid.

Now let us consider the following equation:

$$
x_{j}=U_{j}\left(x_{j}\right)+\sum_{k=1}^{j-1} a_{j k}\left[x_{k}+G_{k}\left(x_{k}\right)\right](j=1,2, \ldots)
$$

where the mappings $G_{k}: C^{n} \rightarrow C^{n}$ have the property Eq. (2) and the mappings $U_{j}: C^{n} \rightarrow C^{n}$ have the property

$$
\left|U_{j}\left(x_{j}\right)\right|_{C^{n}} \leq \sum_{k=1}^{j-1} \alpha_{j k}\left|x_{k}\right|_{C^{n}}, \quad j=1,2, \ldots
$$

and $\alpha_{j k} \geq 0$ are nonnegative numbers.

Rewrite Eq. (20) as

$$
x=U(x)+V x+F(x)
$$

where $U=\left(U_{k}\right), V$, and $F$ are defined in $c_{0}\left(C^{n}\right)$ by

$$
[V x]_{j}=\sum_{k=1}^{j-1} a_{j k} x_{k} \quad \text { and } \quad[F x]_{j}=\sum_{k=1}^{j-1} a_{j k} G_{k}\left(x_{k}\right)
$$

Suppose that

$$
N_{\alpha} \equiv\left[\sum_{j=1}^{\infty} \sum_{k=1}^{j-1}\left|\alpha_{j k}\right|^{2}\right]^{\frac{1}{2}}<\infty
$$

The following result is a particular extension of Lemma 1. 
Theorem 2. Let conditions (2) and (21) hold, with $r=$ $\infty$. Then, under the conditions (4), (6), (23) and

$$
m(V)\left(N_{\alpha}+N_{q}\right)<1
$$

a solution $x=\left(x_{k}\right)$ of $E q .(20)$ is in $l^{2}$. Moreover, it satisfies the inequality

$$
|x|_{l^{2}} \leq m(V)\left[1-m(V)\left(N_{\alpha}+N_{q}\right)\right]^{-1} .
$$

Proof. We have

$$
\begin{aligned}
\left|U_{j}\left(x_{j}\right)\right|_{C^{n}}^{2} & \leq\left(\sum_{k=1}^{j-1} \alpha_{j k}\left|x_{j}\right|_{C^{n}}\right)^{2} \\
& \leq\left(\sum_{k=1}^{j-1}\left|\alpha_{j k}\right|^{2}\right)\left(\sum_{k=1}^{j-1}\left|x_{k}\right|_{C^{n}}^{2}\right) .
\end{aligned}
$$

This yields,

$$
\begin{aligned}
|U|_{l^{2}}^{2} & =\sum_{j=1}^{\infty}\left|U_{j}\left(x_{j}\right)\right|_{C^{n}}^{2} \leq \sum_{j=1}^{\infty}\left[\sum_{k=1}^{j-1}\left|\alpha_{j k}\right|^{2} \sum_{k=1}^{j-1}\left|x_{k}\right|_{C^{n}}^{2}\right] \\
& \leq\left(\sum_{j=1}^{\infty} \sum_{k=1}^{j-1}\left|\alpha_{j k}\right|^{2}\right) \sum_{k=1}^{\infty}\left|x_{k}\right|_{C^{n}}^{2}=N_{\alpha}^{2}|x|_{l^{2}}^{2} .
\end{aligned}
$$

On the other hand,

$$
x=(I-V)^{-1}(U(x)+F(x)) .
$$

Thus,

$$
\begin{aligned}
|x|_{l^{2}} & \leq m(V)\left[|U|_{l^{2}}+|F|_{l^{2}}\right] \\
& \leq m(V)\left[N_{\alpha}|x|_{l^{2}}+N_{q}|x|_{l^{2}}+1\right] .
\end{aligned}
$$

By Eq. (24), it follows that

$$
|x|_{l^{2}} \leq m(V)\left[1-m(V)\left(N_{\alpha}+N_{q}\right)\right]^{-1} .
$$

As claimed.

\section{Acknowledgements}

This research was supported by the Israel Ministry of Science and Technology and by the Fondecyt Chile (Grant No. 1000023)

\section{References}

Crisci, M.R., Kolmanovskii, V.B., Russo, E. and Vecchio, A. (1995) "Stability of continuous and discrete Volterra integro-differential equations by Lyapunov approach", J. Integr. Eq. Appl. 7(4), $393-411$.

Elaydi, S. (1996) An Introduction to Difference Equations (Springer, New York).

Elaydi, S. and Murakami, S. (1996) "Asymptotic stability versus exponential stability in linear Volterra difference equations of convolution type", J. Diff. Eq. 2, 401-410.

Gil',, M.I. (1995) Norm Estimates for Operator-Valued Functions and Applications (Marcel Dekker, New York).

Kolmanovskii, V.B. and Myshkis, A.D. (1998) "Stability in the first approximation of some Volterra difference equations", J. Diff. Eq. Appl. 3, 563-569.

Kolmanovskii, V.B., Myshkis, A.D. and Richard, J.-P. (2000) "Estimate of solutions for some Volterra difference equations", Nonlin. Anal., TMA 40, 345-363.

Kwapisz, M. (1992) "On $l_{p}$ solutions of discrete Volterra equations", Aequationes Mathematicae, University of Waterloo 43, 191-197.

Medina, R. (1996) "Solvability of discrete Volterra equations in weighted spaces”, Dyn. Syst. Appl. 5, 407-422.

Medina, R. (1999) "Stability results for nonlinear difference equations", Nonlin. Stud. 6(1), 73-83. 


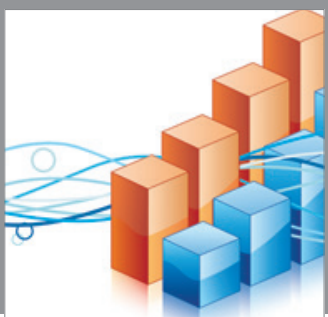

Advances in

Operations Research

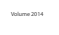

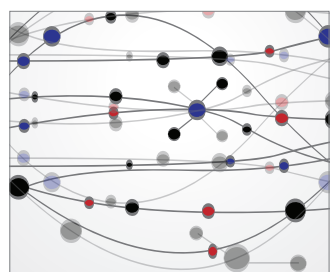

\section{The Scientific} World Journal
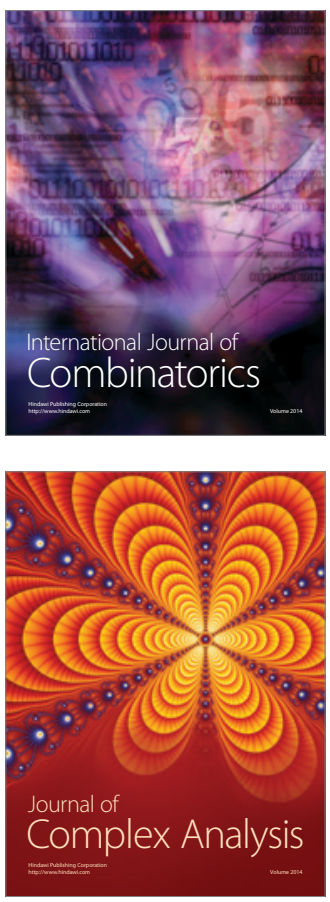

International Journal of

Mathematics and

Mathematical

Sciences
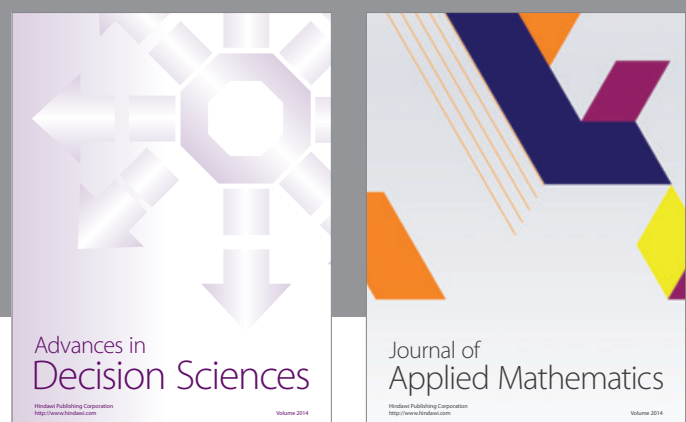

Journal of

Applied Mathematics
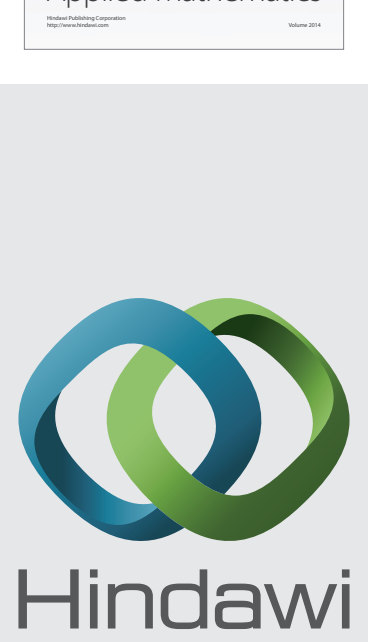

Submit your manuscripts at http://www.hindawi.com
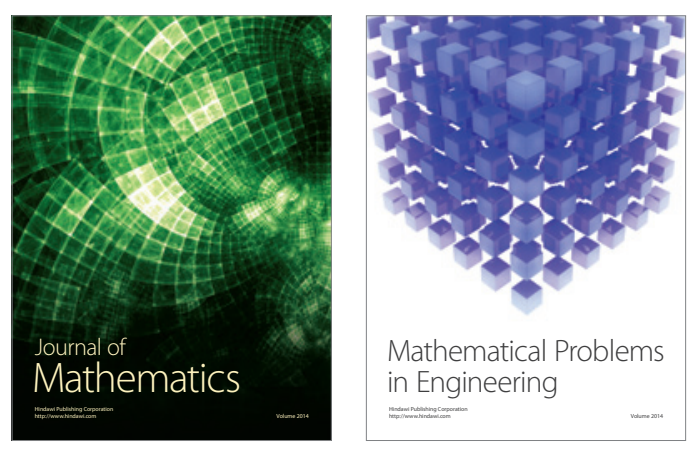

Mathematical Problems in Engineering
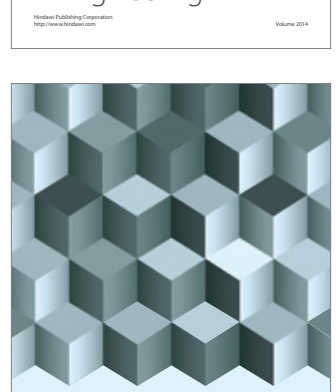

Journal of

Function Spaces
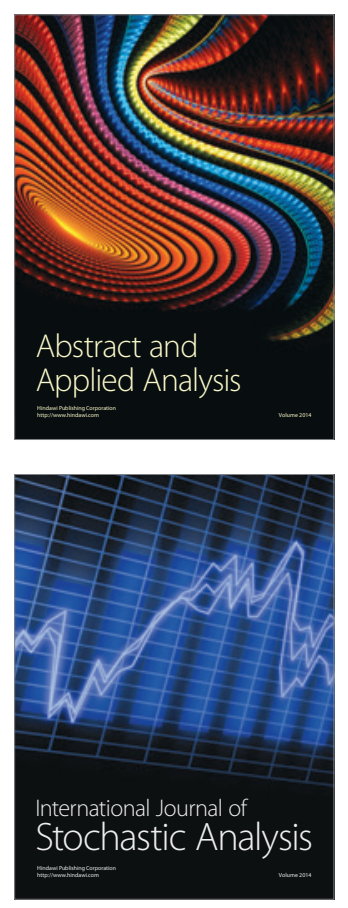

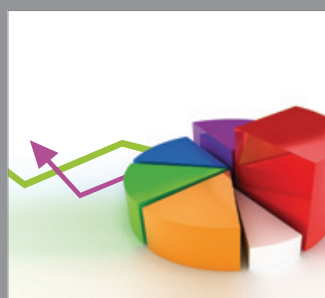

ournal of

Probability and Statistics

Promensencen
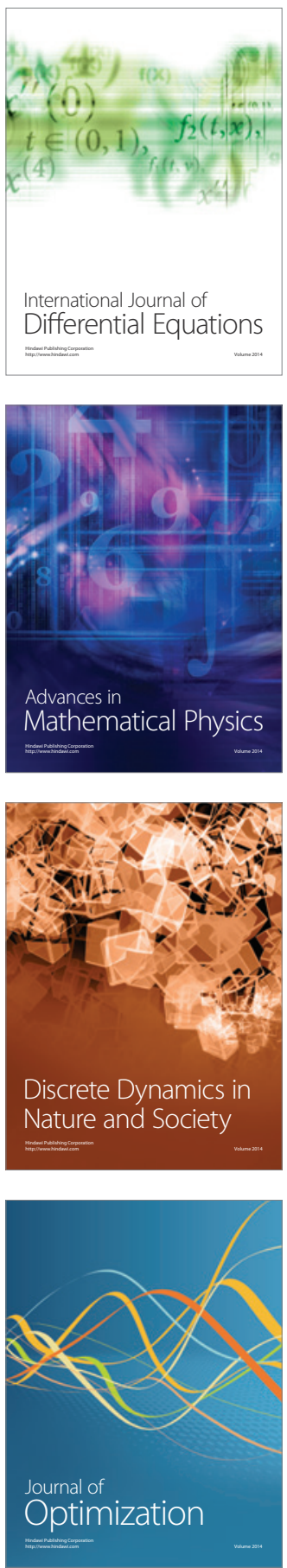